\title{
Applied Theatre in Adolescent Mental Health Transition Care
}

\section{THE TRANSITION FROM CAMHS TO AMHS}

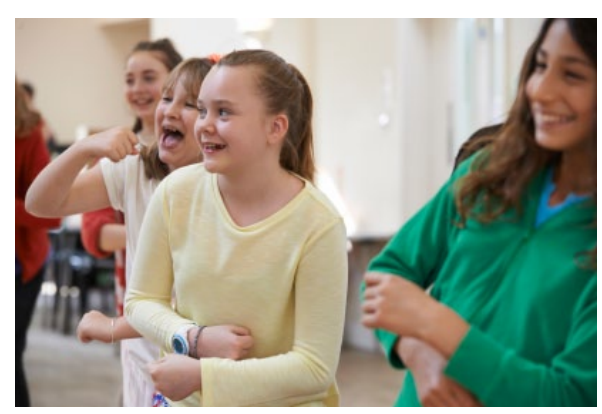

The transition from Child and Adolescent Mental Health Services (CAMHS) to Adult Mental Health Services (AMHS) has been recognised as a key concern within healthcare, described as a 'cliff edge' by the House of Commons Health

Committee, and 'poorly planned, poorly executed and poorly experienced' by the National Institute for Health Research. ${ }^{1,2}$ This short article identifies one way of working in this area, together with plans to conduct research evaluating the initiative.

CAMHS and AMHS are very different services, with distinct histories, philosophies and approaches; adult psychiatry evolved out of the asylum, whereas child psychiatry emerged later and concerned itself more with developmental issues, education and family relationships. For a young person negotiating this interface (usually on turning 18 , although sometimes younger, as services across the country do operate differently), it therefore does not simply involve adjusting to a new physical environment and new professionals, but also adapting to a service that considers them as autonomous adults, rather than as part of the family unit. As well as the level and style of intervention, differences in eligibility thresholds for referral also means that the remit for AMHS is narrower and they may get no input from services at all.

When discussing (or referring to) transition, it is useful to distinguish this from transfer, where the latter is simply the termination of input from child services and the commencement of input from adult services. Transition should be a therapeutic experience and involve preparation: 'a period of handover or joint care, transition planning meetings (involving the young person and carer, and key CAMHS and AMHS professionals) and transfer of case notes or information summaries' ${ }^{3}$ Young people experiencing this quality of transition is, sadly, rare, and thus - while also recognising the need for systemic change, locally and nationally - support for young people negotiating the challenges of moving between services needs to be developed.

\section{WHY APPLIED THEATRE?}

The recent launch of the All-Party Parliamentary Group report on Arts, Health and Wellbeing has highlighted the importance and power of creative approaches to healthcare, ${ }^{4}$ although the relationship between health and wellbeing is not a new phenomenon. Arts-based interventions can support public mental health initiatives by engaging communities and building health literacy.

Applied Theatre is an umbrella term for participatory forms of dramatic activity, where drama and theatre are used in different settings and with diverse groups, for social and therapeutic purposes, and as a vehicle for change. These practices, which can take many forms, do not operate exclusively within health contexts, but often have a focus on the wellbeing of participants, hopefully providing opportunities for selfexpression, reflection and social critique.

So why employ these practices with young people skirting the 'cliff edge' of the transfer of their care from CAMHS to
AMHS? That is the question my research seeks to interrogate, examining if a model of Applied Theatre Practice could build social and emotional capabilities, such as confidence, resilience and selfadvocacy, that might support young people in this transition.

Discourses on drama in mental health contexts and the transition between CAMHS and AMHS have not previously been brought together; yet, if 'young people need to be trained and empowered to become effective partners in their own transition', 5 this is a rich avenue for research and practice. Questions I am exploring in my practicebased research include the following:

- Can role-play and storytelling be used to tackle the external and internalised stigma of mental illness?

- Can the aesthetic detachment of fiction and character facilitate a more self-compassionate approach from young people?

- What impact does creative storytelling and imagining other selfnarratives have on their relationship with the autobiographical engaging communities and 'mental health story' many service users have to

repeatedly tell?

- How can an awareness of our potential 'role repertoire' and of the performative nature of everyday life support transition care and capitalise on the developmental plasticity of adolescence?

Applied Theatre could provide a forum in which to build the interpersonal skills of young people with mental health challenges. Working in the fictional realm also provides an aesthetic distance, which can facilitate heightened objectivity and critical awareness. Dramatic play could interrupt a pattern of fidelity to a 
rigid identity, providing multiple possible identities for young people to align themselves; this demonstrates that the self can be changed and could be a liberating experience.

I am conducting my research by gathering initial qualitative data (through online questionnaires) on the experiences of service users who have been through the transition between CAMHS and AMHS, their parents and carers, and the professionals who work with them. The problems they identify, and the subsequent skills and capacities they feel would be most useful to support them in this transition, will inform the design of my creative practice, which I will then pilot with current service users.

Public mental health has an important role in this context regarding building more resilient communities; however, as with all 'interventions' designed to support service users, practitioners need to ensure that they are not shifting the responsibility of ameliorating the challenges of this transition onto vulnerable young people in place of system change. Supporting service users to better cope with the problems of poor transition needs to happen alongside improvements to the transition between the services themselves, so that the agreed principles of good transitional care are met. ${ }^{6}$ I suggest that if practitioners do not acknowledge the effects and affects of the current problems with traditional service structures, policy and practice, they risk sticking a plaster over a deeper wound. Supporting young people is crucial, but so is changing the system.

\section{FUNDING}

This study was funded by the South West and Wales Doctoral Training Partnership.

\section{Kate Massey-Chase University of Exeter, Exeter, UK}

1. House of Commons Health Committee. Children's and Adolescents' Mental Health and CAMHS: Third Report of the session 20014-15. London: The Stationary Office Ltd, 2014, p. 4.

2. Singh SP Paul M, Islam Z et al. Transition from CAMHS to Adult Mental Health Services (TRACK): A Study of Service Organisation, Policies, Process and User and Carer Perspectives: Report for the National Institute for Health Research Service Delivery and Organisation
Programme. Queen's Printer and Controller of HMSO, 2010, p. 173.

3. Paul M, Ford T, Kramer T et al. Transfers and transitions between child and adult menta health services. The British Journal of Psychiatry 2013; 202(54): 36.

4. All-Party Parliamentary Group on Arts, Health and Wellbeing Inquiry Report. Creative Health: The Arts for Health and Wellbeing, 2017. Available online at: http://www. artshealthandwellbeing.org.uk/appg-inquiry/ (last accessed 9 October 2017).
5. Paul M, Ford T, Kramer T et al. Transfers and transitions between child and adult mental health services. The British Journal of Psychiatry 2013; 202(54): 39.

6. NICE. Transition from Children's to Adults' Services for Young People Using Health or Social Care Services - NICE Guideline, 2016. Available online at: https://www.nice.org.uk/guidance/ ng43/resources/transition-from-childrens-toadults-services-for-young-people-using-healthor-social-care-services-pdf-1837451149765 (last accessed 9 October 2017). 